\title{
Update on Cardiopulmonary Resuscitation Guidelines of Interest to Anesthesiologists
}

\author{
Luiz Fernando dos Reis Falcão ${ }^{1}$, David Ferez ${ }^{2}$, José Luiz Gomes do Amaral ${ }^{3}$
}

\begin{abstract}
Summary: Falcão LFR, Ferez D, Amaral JLG - Update on Cardiopulmonary Resuscitation Guidelines of Interest to Anesthesiologists.
Background and objectives: The new cardiopulmonary resuscitation (CPR) guidelines emphasize the importance of high-quality chest compressions and modify some routines. The objective of this report was to review the main changes in resuscitation practiced by anesthesiologists.

Contents: The emphasis on high-quality chest compressions with adequate rate and depth allowing full recoil of the chest and with minimal interruptions is highlighted in this update. One should not take more than ten seconds checking the pulse before starting CPR. The universal relationship of 30:2 is maintained, modifying its order, initiating with chest compressions, followed by airways and breathing (C-A-B instead of A-B-C). The procedure "look, listen, and feel whether the patient is breathing" was removed from the algorithm, and the use of cricoid pressure during ventilations is not recommended any more. The rate of chest compressions was changed for at least one hundred per minute instead of approximately one hundred per minute, and its depth in adults was changed to $5 \mathrm{~cm}$ instead of the prior recommendation of 4 to $5 \mathrm{~cm}$. The single shock is maintained, and it should be of 120 to $200 \mathrm{~J}$ when it is biphasic; and $360 \mathrm{~J}$ when it is monophasic. In advanced cardiac life support, the use of capnography and capnometry to confirm intubation and monitoring the quality of CPR is a formal recommendation. Atropine is no longer recommended for routine use in the treatment of pulseless electrical activity or asystole.
\end{abstract}

Conclusions: Updating the phases of the new CPR guidelines is important, and continuous learning is recommended. This will improve the quality of resuscitation and survival of patients in cardiac arrest.

Keywords: Cardiopulmonary Resuscitation; Heart Arrest; Heart Massage.

\section{INTRODUCTION}

The knowledge of cardiopulmonary resuscitation (CPR) maneuvers is a priority for all health care professionals ${ }^{1}$. Anesthesiologists constantly deal with critical situations and are prone to witness episodes of cardiac arrest depending on patient's status, surgery, and changes resulting from the anesthetic process. In this environment, current knowledge and quick and precise attitudes determine the prognosis and survival free of sequelae.

The incidence of cardiac arrest during anesthesia varies in the literature ranging from $1: 82,641$ to $1: 2,5002,3$. The main causes are related to changes in physical status $(23.9: 10,000)$

Receives from the Anesthesiology, Pain and Intensive Care Medicine Discipline of the Universidade Federal de São Paulo - Escola Paulista de Medicina (UNIFESP-EPM), Brazil.

1. Post-graduate student of the Anesthesiology, Pain and Intensive Care Medicine Discipline of the UNIFESP-EPM

2. Professor of the Anesthesiology, Pain and Intensive Care Medicine of the UNIFESP-EPM

3. Senior Professor of the Anesthesiology, Pain and Intensive Care Medicine of the UNIFESP-EPM

Submitted on November 8, 2010

Approved on January 31, 2011.

Correspondence to:

Dr. Luiz Fernando dos Reis Falcão

Rua Napoleão de Barros, 715 - 5aadar

Vila Clementino

04024-900 - São Paulo, SP, Brazil

E-mail: luizfernandofalcao@gmail.com followed by surgical complications (4.64:10,000), and isolated anesthetic complications $(1.71: 10,000){ }^{4}$.

In 1960 a group of cardiopulmonary resuscitation pioneers combined mouth-to-mouth resuscitation with chest compressions to create cardiopulmonary resuscitation, the saving method we currently call "CPR". This action when performed immediately after a sudden cardiac arrest can duplicate and even triplicate - the survival chances of a victim. With its evolution, in 1992 the International Liaison Committee on Resuscitation (ILCOR), which meets twice a year to provide a discussion and coordination forum of all aspects of cardiopulmonary resuscitation, was created ${ }^{5}$. On October 18,2010 , it resulted in the last publication with the new recommendations for the care of patients with cardiorespiratory arrest (CRA) and urgent cardiovascular care. The objective of this report was to present in a simplified and objective way the new recommendations for CPR procedures according to the ILCOR guidelines.

\section{BASIC LIFE SUPPORT}

Basic Life Support (BLS) is the pillar of care for patients in cardiorespiratory arrest aiming at maintaining oxygenation and perfusion of vital organs through continuous maneuvers ${ }^{6}$. The fundamental aspects of BLS include the immediate recognition of cardiac arrest and activation of the emergency response team, early CPR, and fast defibrillation. 
According to this new guideline a great emphasis has been given to the quality of care with rapid and strong chest compressions, and defibrillation and advanced care should be given in a way to minimize interruptions in CPR ${ }^{7}$. The first rescuer should rapidly evaluate the presence of cardiac arrest by calling the patient and identifying apnea or respiratory distress, such as gasping ${ }^{8}$. Even with the delay and difficulty of verifying the pulse ${ }^{9}$, the recommendation is still the same for the health care professional, and it should not take more than ten seconds ${ }^{10}$.

Chest compressions consist of rhythmic and strong compressions in the lower half of the sternum, and they should be quickly performed in all cardiac arrest patients ${ }^{11-13}$. They are effective when applied intensely and quickly, with a rate of at least one hundred per minute and at a depth of at least $5 \mathrm{~cm}$. Full chest recoil should be observed after each compression allowing the heart to be completely filled with blood before the next compression. The compression-ventilation ratio of 30:2 has been maintained until the insertion of an advanced airway device, at which point they will become continuous chest compressions (at least one hundred per minute) and one ventilation every six to eight seconds (eight to ten ventilations per minute). Figure 1 shows the complete algorithm of basic life support for a health care professional.
Fatigue of the person performing chest compressions can lead to inadequate rate and depth of chest compressions ${ }^{14-16}$. Significant fatigue with superficial compressions is common after one minute of CPR. When two or more rescuers are available, taking turns every two minutes (or five cycles of compression-ventilation at a 30:2 ratio) is reasonable to prevent a reduction in the quality of compressions. This change should be made during any intervention associated with appropriate interruption in chest compressions (for example during defibrillation). All efforts should be made to make the exchange in less than five seconds. The anesthesiologist should avoid interruptions as much as possible and try to limit then to no more than ten seconds.

In this new guideline a change in the order of beginning CPR is emphasized recommending chest compression before ventilation ( $C-A-B$ instead of $A-B-C$ ). This change reflects the growing evidence of the importance of chest compressions. The second step after high-quality chest compressions begins with opening the airways, which can be performed by hyperestension the neck (head tilt-chin lift maneuver) when evidence of cranial or cervical trauma is lacking. Between $0.12 \%$ and $3.7 \%$ of trauma victims have spinal cord injury ${ }^{17-}$ 19 , and the risk is increased in patients with craniofacial trauma ${ }^{20,21}$, or Glasgow coma scale $<8^{22,23}$, or both ${ }^{21,22}$. If the

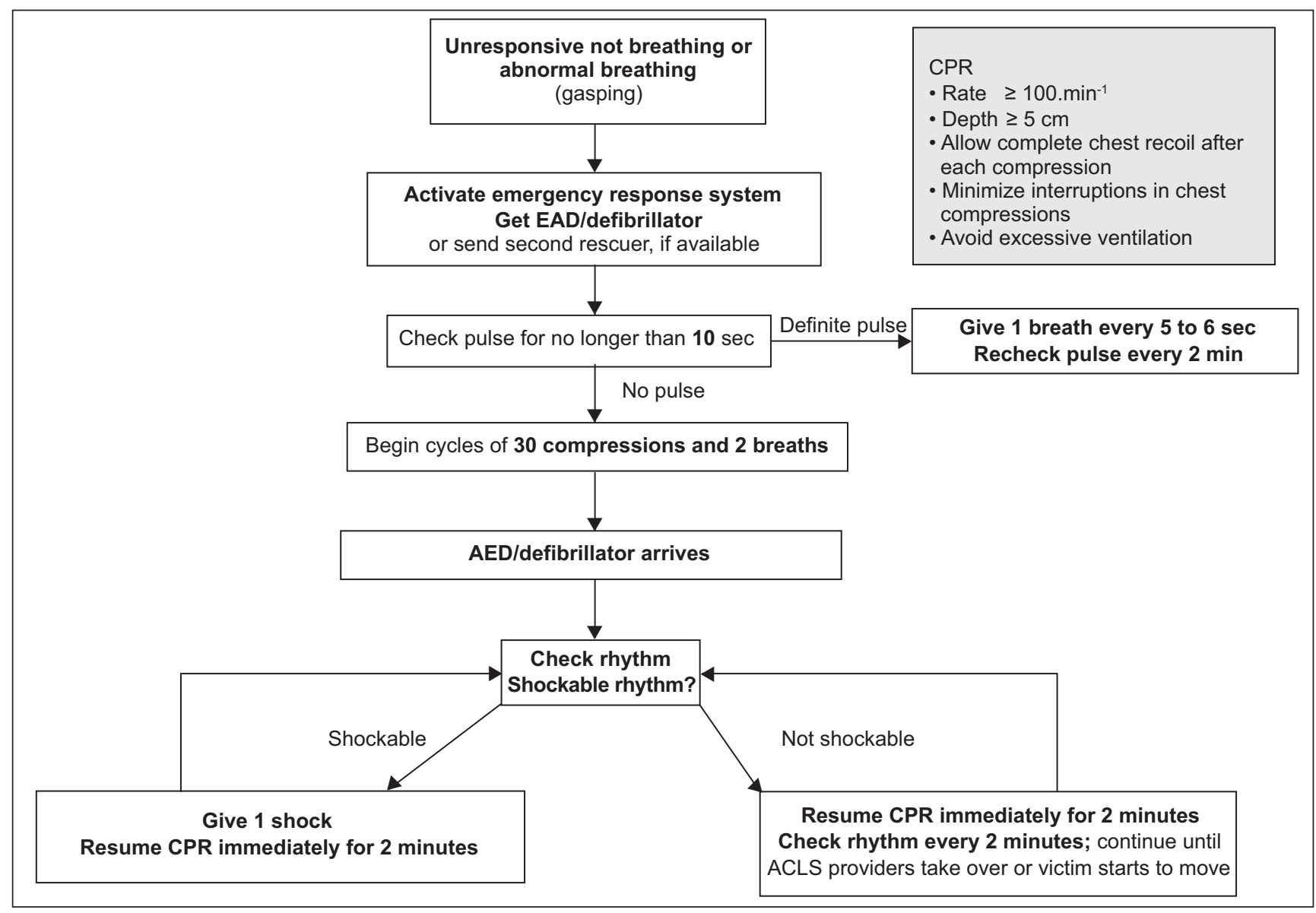

Figure 1 - Basic Life Support Algorithm for Health Care Professionals.

EAD: External Automatic Defibrillator. 
anesthesiologist suspects a cervical spine injury he should open the airways by the anteriorization of the mandible without hyperextending the neck (jaw trust maneuver) ${ }^{24}$; however, if this maneuver is ineffective he should perform the head tilt-chin lift maneuver.

Many of the recommendations for ventilation process contained in the 2005 Guidelines were maintained. During CPR, the primary objective of assisted ventilation is to maintain adequate oxygenation, while the secondary objective is the elimination of $\mathrm{CO}_{2}$. Each ventilation should be performed during one second with a sufficient tidal volume to promote visible thoracic expansion ${ }^{25}$. During CPR the cardiac output is approximately $25 \%$ to $33 \%$ of normal; thus, oxygen and $\mathrm{CO}_{2}$ exchange by the lungs is reduced. As a consequence, low minute volume (lower than the normal tidal volume and respiratory rate) can maintain effective oxygenation and ventilation ${ }^{25-28}$. For this reason, during CPR in adults, a tidal volume of approximately 500 to $600 \mathrm{~mL}$ ( 6 to $7 \mathrm{~mL}^{\mathrm{k}} \mathrm{kg}^{-1}$ ) is enough ${ }^{29-31}$. Excessive ventilation is unnecessary and should be avoided as it causes gastric distension, resulting in complications, such as regurgitation and bronchoaspiration ${ }^{32-34}$. More important, by increasing intrathoracic pressure, decreasing venous return, and reducing cardiac output and survival, it can be harmful ${ }^{34}$. However, there is insufficient research available recommending the ideal tidal volumes, respiratory rate, or fraction of inspired oxygen during resuscitation.

During the first few minutes of a cardiac arrest in ventricular fibrillation (VF), ventilations are not as important as chest compressions ${ }^{35-37}$, since the oxygen in the arterial blood of the patient in cardiorespiratory arrest remains unchanged until the beginning of CPR; then, partial oxygen pressure remains adequate during several minutes of resuscitation. In victims of prolonged cardiac arrest, ventilation and chest compressions are equally important because arterial oxygen is reduced and alveolar pressure of oxygen is insufficient. Ventilation and chest compression are also equally important in patients with cardiac arrest due to asphyxia, drowning, or children in situations of hypoxia ${ }^{38,39}$.

The routine use of cricoid cartilage compression during ventilations is not recommended. Seven randomized, controlled studies have demonstrated that cricoid pressure can delay or avoid the correct placement of an advanced airway device and aspiration can be performed despite of this maneuver $40-46$

\section{ELECTRICAL THERAPY}

Rescuers attending an episode of intrahospital CPR and who have rapid access to a defibrillator should first perform CPR immediately and, as soon as possible, defibrillation. This recommendation is made to reinforce early CPR and defibrillation. When ventricular fibrillation is present for more than a few minutes oxygen and metabolic substrates are depleted. A short period of chest compressions can provide oxygen and energy substrates, increasing the likelihood of a successful defibrilla- tion with return of spontaneous circulation ${ }^{47}$. However, there is not enough evidence supporting or refuting CPR before defibrillation. In monitored patients the time between the diagnosis of VF and defibrillation should be lower than three minutes. When two or more rescuers are present, one starts CPR while the other arranges and prepares the defibrillator.

Recent human studies ${ }^{48,49}$ demonstrated significant survival in patients submitted to a single shock when compared to the use of three shocks and, therefore, the recommendation of a single shock followed by CPR is maintained. The first shock efficacy of biphasic defibrillator is comparable to or better than three shocks of monophasic defibrillator. The consensus for the use of the monophasic defibrillator includes the use of $360 \mathrm{~J}$ in all shocks. The biphasic defibrillator should be charged according to the recommendations of the manufacturer (120 to $200 \mathrm{~J}$ ). If this guidance is unknown the maximal charge should be used.

In pediatric patients data in the literature are insufficient to prove the minimum or maximum safe effective charge. The initial dose of $2{\mathrm{~J} . \mathrm{kg}^{-1}}^{-}$for the monophasic defibrillator has been proven to be effective for reversion in $18 \%$ to $50 \%$ of VF ${ }^{50-52}$ and when a similar charge is used in a biphasic defibrillator it is successful in $48 \%$ of the times ${ }^{52}$. However, even with high energy (above $9 \mathrm{J.kg}$ ), defibrillation is successful without evidence of adverse events ${ }^{53-56}$. Thus, for pediatric

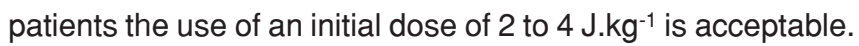
Subsequent defibrillations should have a maximal charge of $4 \mathrm{J.kg}^{-1}$ and an increase in energy can be considered, never

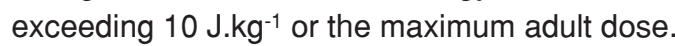

Evidence demonstrate that the four placements of electrodes for defibrillation (anterolateral, anteroposterior, left-anterior and infrascapular, and right-anterior and infrascapular) 57 are equally effective in the treatment of atrial or ventricular arrhythmias ${ }^{58-62}$. Any one of the four positions can be used for defibrillation; however, to facilitate orientation and training, the anterolateral position should be used.

In patients with implantable cardioverter-defibrillator (ICD) or pacemaker, the device has the potential of malfunctioning after defibrillation when the paddles are placed close to the device ${ }^{63,64}$. A study with a cardioverter ${ }^{63}$ demonstrated that the paddles for defibrillation should be at least $8 \mathrm{~cm}$ from the cardioverter to prevent changes in function of the ICD or pacemaker. The anteroposterior and anterolateral positions are acceptable and avoiding placing the paddles on the device to prevent delaying the defibrillation process is recommended.

\section{ADVANCED LIFE SUPPORT}

To treat cardiac arrest, interventions for advanced life support should be preceded by adequate basic life support with early recognition and activation of the emergency response team, and rapid CPR and defibrillation to increase the probability of return of spontaneous circulation (ROSC) with pharmacologic 
therapy and the use of advanced airway monitoring devices. After ROSC, survival and neurological outcome can be improved with specific and systematic post-cardiac arrest care in the ICU with the use of therapeutic hypothermia and reduction of the fraction of inspired oxygen, using the lower level to obtain an arterial oxygen saturation of $94 \%$.

According to the new 2010 guidelines, some key recommendations were added. The use of capnography and capnometry during the peri-CPR period is recommended to confirm tracheal intubation and follow-up the quality of chest compressions. The algorithm has been simplified to emphasize the importance of quality CPR. Atropine is no longer recommended as routine treatment for patients with pulseless electrical activity (PEA) or asystole.

\section{Airway management and ventilation}

The ideal level of the fraction of inspired oxygen $\left(\mathrm{FiO}_{2}\right)$ to be used during the ventilation process of a patient in CRA is not known. Although prolonged exposure to $\mathrm{FiO}_{2}$ of $100 \%$ is potentially toxic, evidence indicating such occurrence in patients submitted to this exposure for a short period of time during CPR is lacking ${ }^{65-67}$. The empirical use of $\mathrm{FiO}_{2}$ of $100 \%$ during CPR optimizing arterial oxygen concentration, therefore increasing $\mathrm{O}_{2}$ delivery, is recommended.

All health care professionals should be trained and qualified to use the bag-valve-mask device during ventilations ${ }^{68,69}$. However, this is not recommended when the professional administers CPR alone and, in this case, mouth-to-mouth or mouth-to-mask is more efficient. When a second rescuer is available, the bag-valve-mask device can be used as long as he has been trained and has experience with this device. The adult bag ( 1 to $2 \mathrm{~L}$ ) should be used, delivering a tidal volume of approximately $600 \mathrm{~mL}$, which is enough to promote thoracic expansion for one second ${ }^{30}$.

Because the upper airway obstruction is secondary to the fall of the tongue, the use of oropharyngeal cannula can improve ventilation. Although studies do not consider specifically the use of the oropharyngeal cannula in patients in CRA, and incorrect placement of this device could worsen the obstruction due to tongue dislocation to the postero-inferior region, the use of this device is recommended in unconscious patients by trained personnel.

The cost-benefit of obtaining an advanced airway should be evaluated during CPR. There are not enough studies indicating the ideal moment for this purpose. In a record of 25,006 intra-hospital CPRs, securing advanced airway early $(<$ five minutes) was not associated with an increase in the return of spontaneous circulation, but it was associated with increased 24-hour survival ${ }^{70}$.

Tracheal intubation was considered the optimal method of airways management in patients in CRA. The tracheal tube keeps the airways patent and allow aspiration of secretions, ventilation with elevated $\mathrm{FiO}_{2}$, refined tidal volume adjust- ments, and an alternative to the administration of specific drugs. Note that with the balloon it is possible to protect the airways against aspirations. Although intubation can be performed during chest compressions, it is often associated with interruptions of compressions for several seconds. The use of supraglottic devices represents an alternative to tracheal intubation, being recommended in this new guideline. When compared to the face mask, the laryngeal mask (LM) promotes more safe and reliable ventilation ${ }^{71,72}$. Although LM does not guarantee absolute protection against aspiration, studies have revealed that regurgitation is lower with LM than with the face mask, and aspiration is uncommon. Compared to the tracheal tube, LM promotes equivalent ventilation ${ }^{72,73}$, and the reported success rate of ventilation during CPR ranges from $72 \%$ to $97 \%$ of patients 74,75 . For qualified health care professionals the use of the LM is an acceptable alternative to bag-valve-mask ventilation or tracheal intubation for airway management of patients in cardiac arrest.

Once the advanced device has been inserted in the airway it should be evaluated to guarantee the correct positioning. In a retrospective study tracheal intubation was associated with an incidence of $6 \%$ to $25 \%$ of undiagnosed incorrect positioning or dislocation of the tube ${ }^{76}$. This assessment will be done during CPR maneuvers through the physical exam (bilateral chest expansion, and lung and epigastric auscultation), besides identification of expired $\mathrm{CO}_{2}$ and capnography curves. Studies with capnography to determine the tracheal tube placement in patients in CRA have shown $100 \%$ sensitivity and specificity for the correct diagnosis 76,77 . Thereafter, ventilations and compressions will be performed uninterruptedly (eight to ten ventilations.minute ${ }^{-1}$ and one hundred compressions.minute ${ }^{-1}$ ) unless ventilation is inadequate when compressions are not interrupted.

\section{Cardiac arrest management}

Cardiac arrests are associated with four different rhythms: ventricular fibrillation (VF), pulseless ventricular tachycardia (PVT), pulseless electrical activity (PEA), and asystole. Ventricular fibrillation represents a disorganized electrical activity, while PVT is an organized electrical activity, both without the ability to generate blood flow. Pulseless electrical activity comprehends a heterogeneous group of organized electrical rhythm associated with the absence or ineffectiveness of ventricular mechanical activity. Asystole represents the absence of detectable electrical ventricular activity. Figure 2 shows the complete algorithm of the advanced life support.

Understanding the importance of the diagnosis and treatment of the underline cause is fundamental for the management of all rhythms associated with cardiac arrest. During CPR, one should consider the "Hs" and "Ts" to identify and treat the factors responsible for the arrest or that are hindering a successful resuscitation (Table I). 


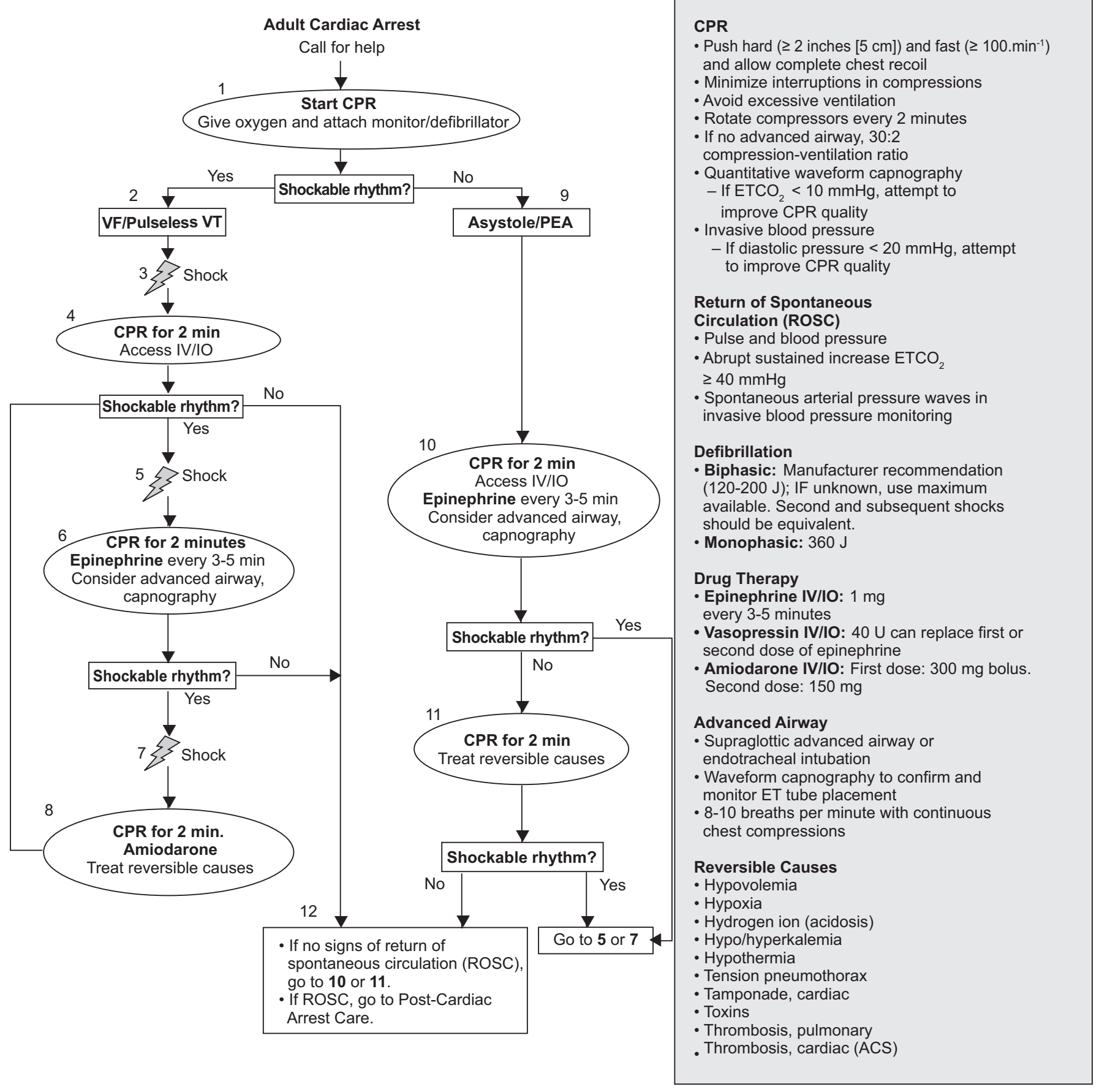

Figure 2 - Advanced Cardiac Life Support Algorithm.

Table I - Treatable Causes of Cardiac Arrest: The H's e T's

\begin{tabular}{ll}
\hline \multicolumn{1}{c}{ H's } & \multicolumn{1}{c}{ T's } \\
\hline Hypoxia & Toxins \\
Hypovolemia & Tamponade (cardiac) \\
Hydrogen ion (acidosis) & Tension pneumothorax \\
Hypo/Hyperkalemia & Thrombosis, pulmonary (embolism) \\
Hypothermia & Thrombosis, coronary (ACS) \\
\hline
\end{tabular}

\section{Ventricular fibrillation and pulseless ventricular tachycardia}

Whenever the diagnosis of VF or PVT is made, one should start immediately chest compressions while a second rescuer charges the defibrillator. The time interval without CPR for defibrillation should be as short as possible with synchronized 
team work. If a biphasic defibrillator is available the charge indicated by the manufacturer (120 or $200 \mathrm{~J}$ ), or the maximum charge, should be used. In case of a monophasic defibrillator, a charge of $360 \mathrm{~J}$ should be used. After the shock, chest compressions should resume (without verifying rhythm or pulse) for two minutes. After this period the sequence is repeated beginning by checking the rhythm.

A precordial thump should be considered only on monitored unstable ventricular tachycardia and when a defibrillator is not available, and it should not delay the onset of CPR and defibrillation.

Whenever VF or PVT persists after the first shock and after two minutes of CPR it is possible to use a vasopressor to increase myocardial blood flow. The maximal effect of a bolus of intravenous (IV) / intraosseous (IO) vasopressor agent is one to two minutes. If the shock fails to reverse the rhythm, a vasopressor should be used to optimize the potential impact of increased myocardial blood flow before the next defibrillation. However, if the shock results in a rhythm that generates a pulse the dose of vasopressor administered before checking the rhythm and pulse can in theory generate a harmful effect in cardiovascular stability. This complication can be avoided by using continuous monitoring, such as capnography, invasive blood pressure, and central venous saturation for the early detection of the return of spontaneous circulation (ROSC) during chest compressions ${ }^{78-80}$. After the shock, pauses to verify the rhythm and pulse should be added; before the administration of the vasopressor, reducing myocardial perfusion during a critical period after the shock can reduce the chances of ROSC.

It has been clinically proven that amiodarone is the first line antiarrhythmic drug in cardiac arrest, since it increases the rate of ROSC. It is indicate in patients with VF/PVT unresponsive to CPR, defibrillation, and vasopressors. If amiodarone is not available, one might consider lidocaine; however, clinical studies did not show increase in the rate of ROSC with lidocaine when compared to amiodarone.

Diagnosis and treatment of the causative factors of VF/PVT are fundamental for the correct management of all rhythms of CRA. Anesthesiologists should not forget the $5 \mathrm{Hs}$ and $5 \mathrm{Ts}$ to identify the causes of CRA or factor that may be complicating the process of resuscitation.

\section{Pulseless electrical activity and asystole}

When verifying the rhythm of cardiac arrest and there is no indication for electrical shock, chest compressions should be immediately initiated and continued for two minutes until a new verification of the rhythm. When this rhythm is organized, one should check the pulse. If a pulse is present, post-cardiac arrest care should be immediately initiated.

A vasopressor should be administered as soon as possible to increase myocardial and cerebral blood flow during CPR and to achieve ROSC. Available evidence suggests that routine use of atropine during PEA or asystole has no benefits.
For this reason, atropine was removed from the new CPR guidelines.

Frequently, pulseless electrical activity is caused by reversible conditions that can be successfully treated if they are correctly identified. During the two minutes of chest compressions, anesthesiologists should remember the $5 \mathrm{Hs}$ and $5 \mathrm{Ts}$ (Table I) that could have led to CPR and correct them.

Asystole is frequently a final rhythm after prolonged VF or PEA and, therefore, the prognosis is frequently very poor.

\section{Monitoring during CPR}

According to the new 2010 guidelines, there is greater emphasis on physiologic monitoring to optimize the quality of CPR and for early detection of ROSC. Animal and human studies indicate that following-up the expired fraction of $\mathrm{CO}_{2}\left(\mathrm{ETCO}_{2}\right)$, coronary perfusion pressure (CPP), and central venous oxygen saturation $\left(\mathrm{SvCO}_{2}\right)$ provide useful information on CPR and patient's response to treatment. In addition, a sudden increase in any of these parameters is a sensitive indicator of ROSC, which can be monitored without interrupting chest compressions 80,81 .

Although it is common practice, studies validating pulse palpation during CPR are lacking. The inferior vena cava does not have valves, and the retrograde blood flow during CPR can produce pulsations in the femoral vein ${ }^{82}$. Carotid pulse during CPR does not show the efficacy of myocardial or cerebral perfusion.

The use of continuous capnography and capnometry during the peri-CPR period is a formal recommendation. With constant ventilation, $\mathrm{ETCO}_{2}$ has good correlation with cardiac output during CPR. This correlation could be altered with the administration of intravenous sodium bicarbonate ${ }^{83}$. This is due to the fact that bicarbonate is converted into $\mathrm{CO}_{2}$ and water, causing a transient increase in the elimination of $\mathrm{CO}_{2}$ by the lungs, and this change should not be mistakenly interpreted as a sign of ROSC. On the other hand, administration of vasopressor causes a transient reduction in $\mathrm{ETCO}_{2}$ due to the increase in afterload and reduction in cardiac output, which should not be interpreted as a reduction in the quality of $\mathrm{CPR}^{84}$. The presence of persistently low $\mathrm{ETCO}_{2}(<10 \mathrm{mmHg})$ during CPR in intubated patients suggests that ROSC is unlikely 78,85 . A study showed that low values of $\mathrm{ETCO}_{2}$ in nonintubated patients during CPR are not a reliable predictor to achieve ROSC ${ }^{86}$. Leaks during ventilation with a face mask or supraglottic device may result in low $\mathrm{ETCO}_{2}$. If $\mathrm{ETCO}_{2}$ is $<10 \mathrm{mmHg}$ in patients being ventilated without leaks, one should improve the quality of CPR with optimization of chest compressions. If a sudden increase in $\mathrm{ETCO}_{2}$ to normal levels ( 35 to $40 \mathrm{mmHg}$ ) is observed, it should be considered an indication of ROSC.

Coronary perfusion pressure (aortic diastolic pressure right atrial diastolic pressure) during CPR correlates with myocardial blood flow and ROSC ${ }^{87}$. A study in humans revealed that ROSC is related with a CPP $\geq 15 \mathrm{mmHg}$ during CPR ${ }^{88}$. The specific target for diastolic blood pressure to optimize the chances of ROSC has not been established. Diastolic blood pressure should be used in monitoring the quality of CPR, opti- 
mizing chest compressions and guiding vasopressor therapy. If diastolic blood pressure is $<20 \mathrm{mmHg}$, one should attempt to improve the quality of compressions with vasopressor, or both. Invasive blood pressure monitoring may also be used in the early detection of ROSC during chest compressions.

Patients being monitored with continuous $\mathrm{SvcO}_{2}$ show during CPR levels of $25 \%$ to $35 \%$ (normal of $60 \%$ to $80 \%$ ) revealing inadequate blood flow. In a clinical study, the presence of $\mathrm{SvCO}_{2}<30 \%$ during CPR was associated with lack of successful ROSC ${ }^{89}$. For this reason, if $\mathrm{SvcO}_{2}$ is lower than $30 \%$, improving resuscitation maneuvers is recommended.

The use of echocardiogram during cardiac arrest has not been specifically investigated regarding its impact on the outcome. However, some studies suggest that transthoracic and transesophageal echocardiogram is potentially useful in the diagnosis and treatment of the causes of cardiac arrest, such as cardiac tamponade, pulmonary embolism, ischemia, and dissection of the aorta ${ }^{90-92}$.

\section{Pharmacologic therapy}

The primary objective of pharmacologic therapy during cardiac arrest is to facilitate recovery and maintenance of spontaneous rhythm with perfusion. With this objective the use of drugs is associated with an increase in ROSC, but not with an increase in long-term survival with good neurologic outcome.

Adrenaline has beneficial effects in patients in cardiac arrest by stimulating alpha-adrenergic receptors, and increasing coronary perfusion pressure and cerebral perfusion pressure ${ }^{93,94}$. The dose of $1 \mathrm{mg} E \mathrm{E} / \mathrm{IO}$ is administered every three to five minutes during CPR. Higher doses may be used when indicated to treat specific problems, such as overdose of beta blockers or calcium channel blockers, or when invasive hemodynamic monitoring is available. If intravenous or intraosseous access is not available, 2 to $2.5 \mathrm{mg}$ of adrenaline may be administered endotracheally, followed by $10 \mathrm{~mL}$ of sterile saline.

Randomized clinical assays and metanalysis did not demonstrate differences in the results (ROSC, survival, and neurologic outcome) with the use of $40 \mathrm{U}$ of IV vasopressin versus $1 \mathrm{mg}$ of IV adrenaline ${ }^{95}$. For this reason, a dose of $40 \mathrm{U}$ of IV/IO vasopressin can substitute the first or second dose of adrenaline in the treatment of cardiac arrest.
Evidence that any antiarrhythmic medication administered routinely during cardiac arrest increases survival is lacking. However, it has been demonstrated that amiodarone increases short-term survival when compared to placebo or lidocaine, and its use should be considered in cases of VF/PVT unresponsive to $\mathrm{CPR}$, defibrillation, and vasopressor therapy at an initial dose of $300 \mathrm{mg} \mathrm{IV/IO} \mathrm{followed} \mathrm{by} \mathrm{a} \mathrm{dose} \mathrm{of} 150 \mathrm{mg}$ $\mathrm{IV} / \mathrm{IO}$, if needed. There is no evidence that supports the use of lidocaine in patients with refractory VF/PVT, and it should only be used in the absence of amiodarone at an initial dose of 1.0 to $1.5 \mathrm{mg} \cdot \mathrm{kg}^{-1} \mathrm{IV}$ and additional doses of 0.5 to $0.75 \mathrm{mg} \cdot \mathrm{kg}^{-1}$ IV with ten-minute intervals and a maximal dose of $3 \mathrm{mg} \cdot \mathrm{kg}^{-1}$. Magnesium sulfate in cardiac arrest is not routinely recommended, but only in the presence of torsades de pointes at a dose of 1 to $2 \mathrm{~g} \mathrm{IV/IO} \mathrm{diluted} \mathrm{in} 10 \mathrm{~mL}$ of D5W.

Clinical studies have demonstrated conflicting evidence regarding the benefits of the routine use of atropine in cardiac arrest 96,97 . For this reason atropine was removed from the asystole and PVT protocol in this last review.

It has been observed that sodium bicarbonate during cardiac arrest is related to a series of adverse events, compromising coronary perfusion pressure by reducing systemic vascular resistance ${ }^{98}$. Furthermore, it promotes extracellular alkalosis, hypernatremia, excess of $\mathrm{CO}_{2}$, and paradoxal intracellular acidosis ${ }^{99}$. In special resuscitation situations, such as preexisting metabolic acidosis, hyperkalemia, or overdoses with tricyclic antidepressants, bicarbonate may be beneficial. However, the routine use of bicarbonate in patients in cardiac arrest is not recommended. Thus, for lack of evidence, calcium is not recommended in CPR ${ }^{100}$.

\section{CONCLUSION}

In cardiac arrests, high-quality cardiopulmonary resuscitation is fundamental for the successful return of spontaneous circulation. During resuscitation, frequent chest compression of adequate depth allowing the complete recoil of the thorax after each compression, minimizing interruptions and avoiding excessive ventilation should be the goals. The quality of CPR should be continuously monitored to optimize resuscitation efforts and early recognition of the return of spontaneous circulation. We hope that this update of the new CPR guidelines improves the quality of resuscitation and survival of patients in cardiac arrest. 